\title{
SAÚDE E DESENVOLVIMENTO SUSTENTÁVEL: UMA ANÁLISE DA CONVERGÊNCIA DAS PUBLICAÇÕES CIENTÍFICAS ${ }^{1}$
}

\author{
Mateus Dias Antunes ${ }^{2}$ \\ Sonia Maria Marques Gomes Bertolini ${ }^{3}$ \\ Rute Grossi Milani ${ }^{4}$ \\ Márcia Aparecida Andreazzi ${ }^{5}$
}

Resumo: Devido a atual preocupação mundial com o alcance dos Objetivos do Desenvolvimento Sustentável, objetivando vidas sustentáveis e saudáveis, torna-se relevante evidenciar a situação e a direção dos estudos nessa área. Este trabalho analisou a consonância das publicações científicas, indexadas em bases de dados mundiais, num período de 25 anos, sobre o tema Saúde e Desenvolvimento Sustentável. Verificou-se um aumento na quantidade de publicações a partir de 2012, com grande diversidade de eixos temáticos. Porém, temas como segurança alimentar, agricultura sustentável, cidades sustentáveis e saudáveis, sociedades pacíficas e inclusivas e educação ambiental foram pouco explorados, evidenciando a necessidade de maior articulação entre esses temas e as ações em saúde, na busca pelo desenvolvimento sustentável e saudável.

Palavras-chave: Estudos Científicos; Meio Ambiente; ODS; Saúde Verde.

${ }^{1}$ Data de Submissão: 08/08/2019 - Data de Aceitação: 07/02/2020

2 Universidade de São Paulo. E-mail: manteusantunes@usp.br, Link para o Lattes: http://lattes.cnpq.br/4926064696686266

3 Universidade Cesumar - Unicesumar / Instituto Cesumar de Ciência, Tecnologia e Inovação. E-mail: sonia.bertolini@unicesumar.edu.br , Link para o Lattes: http://lattes.cnpq.br/9950674050703908

${ }^{4}$ Universidade Cesumar - Unicesumar /Instituto Cesumar de Ciência, Tecnologia e Inovação.

E-mail: rute.milani@unicesumar.edu.br, Link para o Lattes: http://lattes.cnpq.br/8844448878404124

5 Universidade Cesumar - Unicesumar / Instituto Cesumar de Ciência, Tecnologia e Inovação.

E-mail: marcia.andreazzi@unicesumar.edu.br , Link para o Lattes: http://lattes.cnpq.br/0356767742666814 


\begin{abstract}
Due to the current worldwide concern with the achievement of the Sustainable Development Goals, aiming at sustainable and healthy lives, it becomes relevant to highlight the situation and the direction of studies in this area. This work analyzed the consonance of scientific publications, indexed in world databases, over a period of 25 years, on the theme of Health and Sustainable Development. There has been an increase in the number of publications since 2012, with a great diversity of thematic axes. However, topics such as food security, sustainable agriculture, sustainable and healthy cities, peaceful and inclusive societies and environmental education were little explored, highlighting the need for greater articulation between these themes and health actions, in the search for sustainable and healthy development.
\end{abstract}

Keywords: Scientific studies; Environment; ODS; Green Health.

\title{
Introdução
}

A percepção de que a ação humana produz efeitos negativos sobre 0 meio ambiente, provocando desequilíbrios é extremamente recente, datando do final da década de 1960, com as denúncias sobre a poluição provocada a partir da Revolução Industrial, por isso, no final do século XX, as discussões sobre os cuidados com o meio ambiente se tornaram primordiais (PADILHA, 2010).

Desta forma, em 1968 foi convocada a Conferência das Nações Unidas Sobre o Meio Ambiente Humano, que aconteceu em Estocolmo, em 1972, e se tornou um marco histórico. À esta Conferência, deve-se a criação do Programa das Nações Unidas para o Meio Ambiente (PNUMA) e, esse programa, juntamente com a Organização Meteorológica Mundial (OMM), criaram o Painel Intergovernamental de Mudanças Climáticas (IPCC), com o propósito de estudar os efeitos de tais mudanças, seus impactos ambientais e socioeconômicos, e desenvolver estratégias de enfrentamento para a situação. Padilha (2010) afirma que, naquele momento histórico, estava claro que proteger e respeitar o meio ambiente não era somente uma questão ideológica, mas de sobrevivência dos seres humanos.

Dessa maneira, a Comissão Mundial sobre o Meio Ambiente e Desenvolvimento (CMMAD), em 1983, elaborou um relatório intitulado "Nosso Futuro Comum", que discutiu sobre o desenvolvimento e o meio ambiente e que apresentou, pela primeira vez, a proposta de desenvolvimento sustentável, definindo-o como um processo que permite satisfazer as necessidades da população atual, sem comprometer a capacidade de atender as gerações futuras (CMMAD, 1991).

A partir desse momento, outros eventos aconteceram no planeta, como a Rio/92, a aprovação da Agenda 21, a Convenção sobre a Biodiversidade Biológica (CDB) em 1993, e a Cúpula do Milênio, em 2000, que inspiraram os Objetivos do Desenvolvimento do Milênio (ODM) até 2015. Buss et al. (2014) afirmaram que a saúde teve importante presença nos ODM (2000-2015), constituindo três dos oito objetivos, porém, os autores enfatizam que outros ODM, como a sustentabilidade ambiental, também geram impacto sobre a saúde, pois atuam diretamente sobre alguns determinantes socioambientais.

revista brasileira educação ambiental 
Finalizando o período de vigência dos ODM, foi apresentado um relatório para a elaboração dos Objetivos do Desenvolvimento Sustentável (ODS). Os ODS foram definidos pelas Nações Unidas e compõem uma agenda global, adotada em setembro de 2015, contendo 17 objetivos e 169 metas para serem atingidas até 2030. De modo geral, os ODS envolvem ações para acabar com a pobreza, promover a prosperidade e o bem-estar para todos, proteger o meio ambiente e enfrentar as mudanças climáticas (UNESCO, 2019). Nos ODS observa-se que os objetivos relacionados à saúde caminham integrados aos de meio ambiente, apresentando em comum, a busca do desenvolvimento sustentável e saudável, ou seja, o desenvolvimento sem comprometer a saúde do planeta e dos seres humanos.

Considerando a preocupação com a relação entre a saúde das pessoas e o desenvolvimento sustentável, analisar e conhecer a atual situação das publicações referentes a este tema é pertinente. Desta forma, o objetivo deste trabalho foi explorar, na literatura científica mundial, publicações a respeito da relação entre a saúde das pessoas e o desenvolvimento sustentável, buscando entender o comportamento científico dessa consonância, bem como, evidenciar os diferentes segmentos, contribuições e lacunas dos estudos.

\section{Metodologia}

O objeto de análise foi a produção científica veiculada em periódicos indexados nas bases de dados: Scientific Eletronic Library Online (SciELO), acessado pelo site http://www.scielo.org; United States National Library of Medicine (PubMed), acessado pelo site http://www.ncbi.nlm.nhi.gov/pubmed; Web of Science, acessado pelo site https://apps.webofknowledge.com/ e Science Direct, acessado pelo site http://www.sciencedirect.com/.

A busca dos artigos científicos compreendeu um período de 25 anos, avaliando artigos científicos publicados entre os anos de 1992 a 2016. Foram utilizados os descritores em português "saúde e desenvolvimento sustentável" e seu correspondente em inglês "health and sustainable development". Descartando-se as publicações duplicadas e sob a forma de editoriais, comentários, carta ao editor, dentre outros, obteve-se um total de 172 artigos científicos eleitos para este estudo (Figura 1, próxima página).

Após a avaliação dos 172 artigos, que atenderam aos critérios de seleção e análise crítica, foram identificadas e coletadas as seguintes informações: ano de publicação, que permitiu analisar a evolução histórica das publicações, eixo temático central, local de origem da pesquisa e principais periódicos citados com suas respectivas classificações, segundo o critério Qualis/ CAPES/ 2016, na área de Saúde Coletiva.

Os dados foram tabulados, organizados em planilha eletrônica e analisados de forma descritiva. 


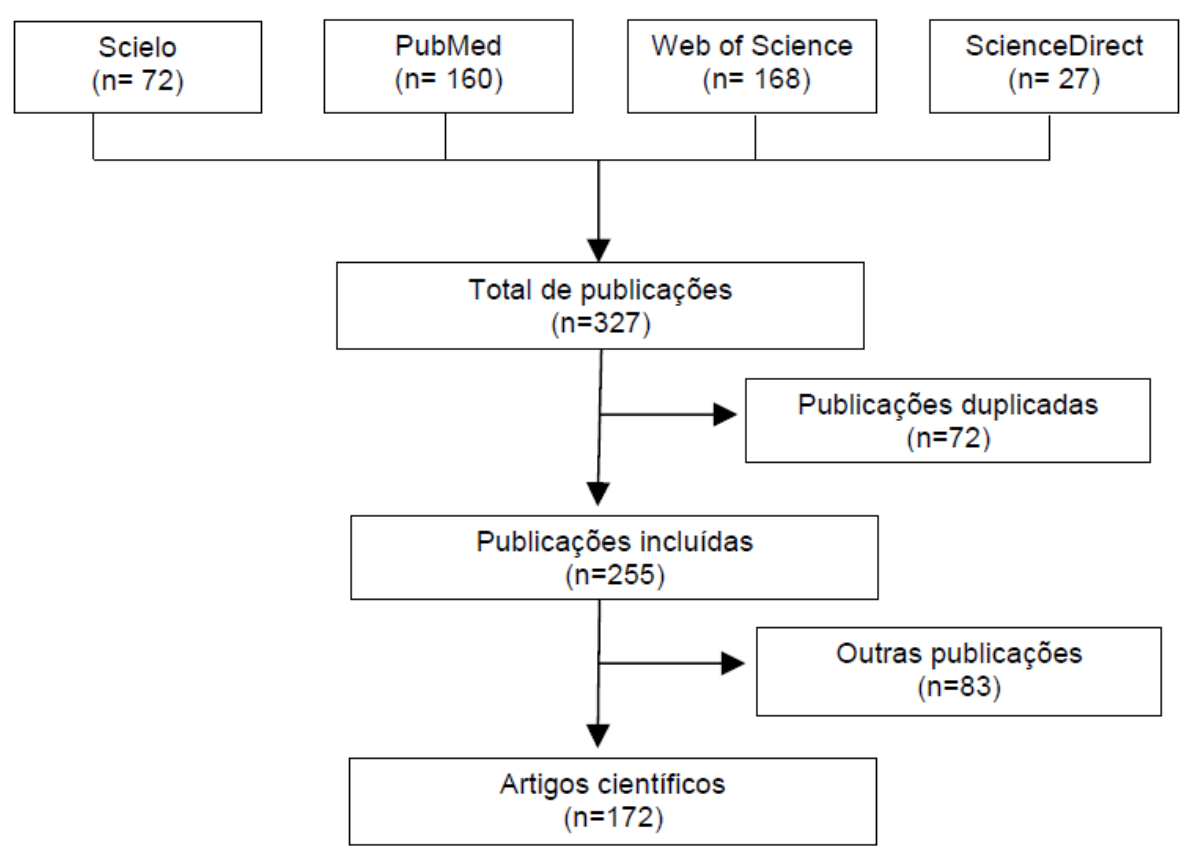

Figura 1: Fluxograma da seleção dos artigos científicos avaliados.

Fonte: elaborado pelos autores.

\section{Resultados e Discussão}

Com relação à evolução histórica, o estudo permitiu identificar o número de artigos científicos nas bases estudadas de acordo com o ano de publicação, no período compreendido entre os anos de 1992 a 2016, evidenciando que a primeira publicação sobre o tema Saúde e Desenvolvimento Sustentável datou de 1992 e, nos anos seguintes, o número de publicações apresentou um comportamento constante até meados de 2012, quando aumentou de forma expressiva (Figura 2).

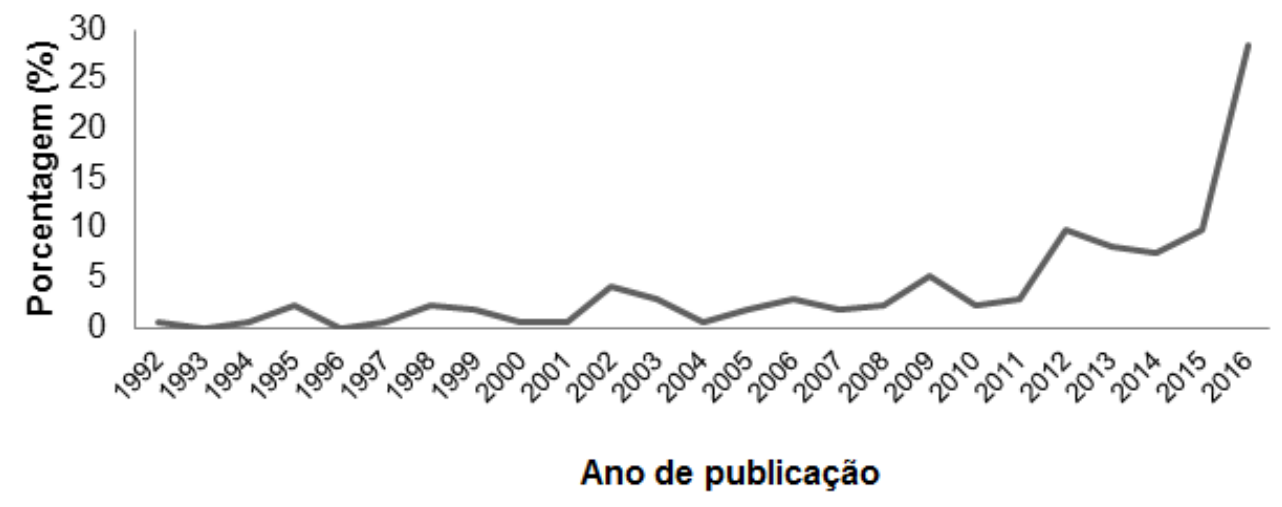

Figura 2: Porcentagem de artigos científicos publicados, sobre saúde e desenvolvimento sustentável, entre os anos de 1992 e 2016.

Fonte: elaborado pelos autores. 
Nesta avaliação histórica, observou-se que Bos (1992) estudou os problemas originados da interface entre ambiente e desenvolvimento que necessitavam ser abordados no contexto do controle de vetores, visto que, segundo o autor, a exploração dos recursos naturais e as mudanças demográficas colocaram vários vetores como fatores biológicos de risco no ambiente para a saúde humana.

Na sequência, Melo e Costa (1994) analisaram o processo de formação de agendas com ênfase na mudança do paradigma conceitual que informa a atuação das agências internacionais na área da saúde e discutiram os paradigmas da saúde pública e da economia da saúde. Prosseguindo no tempo, os trabalhos versaram sobre economia (WARFORD, 1995), reforma do setor de saúde (BERMAN, 1995), agricultura (NELSON, 1995), turismo em saúde (HELLEN, 1995), clima (MARTENS et al., 1997) e cidades (PRICE; TSOUROS, 1997).

Anos mais tarde, Waterston e Lenton (2000) estudaram a relação entre o desenvolvimento sustentável, alterações climáticas globais induzidas pelo homem e a saúde das crianças. Já em 2002, foi possível observar um pequeno aumento no número de publicações que versaram sobre educação ambiental (RIBEIRO et al., 2002); saúde (BRUNDTLAND, 2002); saúde pública (PEIRY, 2002); economia (GIDDINGS et al., 2002); alimentação (ALLI et al., 2002; VORSTER, 2002); perspectivas na Índia (JOHN; ABEL, 2002) e desenvolvimento sustentável na prevenção da delinquência sexual, saúde pública e segurança (VAN LOON, 2002).

Contudo, a partir de 2012 o aumento no número de publicações mostrou uma vertiginosa curva ascendente, totalizando $66,86 \%$ (115/172) das publicações. Ressalta-se que dessa porcentagem, $57,39 \%$ (66/115) são decorrentes dos últimos dois anos avaliados (2015-2016).

Mais uma vez, o que se observa é uma multiplicidade de focos relacionados a saúde e desenvolvimento sustentável, dentre ele destacamos aspectos relacionados à economia e cuidado ecológico (SIGNORINO, 2011; BACKES, 2011), economia verde (GALLO et al., 2012), alterações climáticas, saúde do trabalhador e das crianças, gestão sustentável, desenvolvimento e saúde mental (DESSEILLES et al., 2013; ABOUETTAHIR et al., 2013; RIBEIRO et al., 2013; TOMLINSON, 2013), saúde sexual e reprodutiva, saúde verde (NEWMAN et al., 2014; KHETANI et al. 2014; BOTCHWEY et al., 2014), discussões sobre os ODS, estudos sobre os determinantes sociais de saúde, saúde da mulher, crianças e adolescentes (BROLAN; HILL, 2015; ANDRADE et al., 2015; KIM; EVANS, 2015; GROVE et al., 2015), agricultura familiar, temas relacionados à indústria farmacêutica e diversos trabalhos sobre os objetivos globais para o desenvolvimento sustentável (BORGES, et al. 2016; DELGADO, 2016; FURUKAWA et al., 2016; VALENTINE et al., 2016, CHAPMAN, 2016; BUNDHAMCHAROEN et al., 2016; GALVÃO et al., 2016). 
Com relação à definição dos eixos temáticos, após análise dos artigos, baseados em seus títulos e resumos, definiram-se 38 eixos temáticos, mostrando uma grande variação quanto aos objetivos das pesquisas (Figura 3). Observa-se pela nuvem de palavras que temas como: Objetivos do Desenvolvimento Sustentável (ODS), Pós-2015, Saúde e Meio Ambiente, Saúde Pública e Gestão constituíram as discussões de destaque e mesmo com uma grande variedade de eixos temáticos, notou-se que objetivos como segurança alimentar, agricultura sustentável, acesso à energia, cidades sustentáveis e saudáveis, sociedades pacíficas e inclusivas e educação ambiental apresentaram reduzida articulação com as ações em saúde.

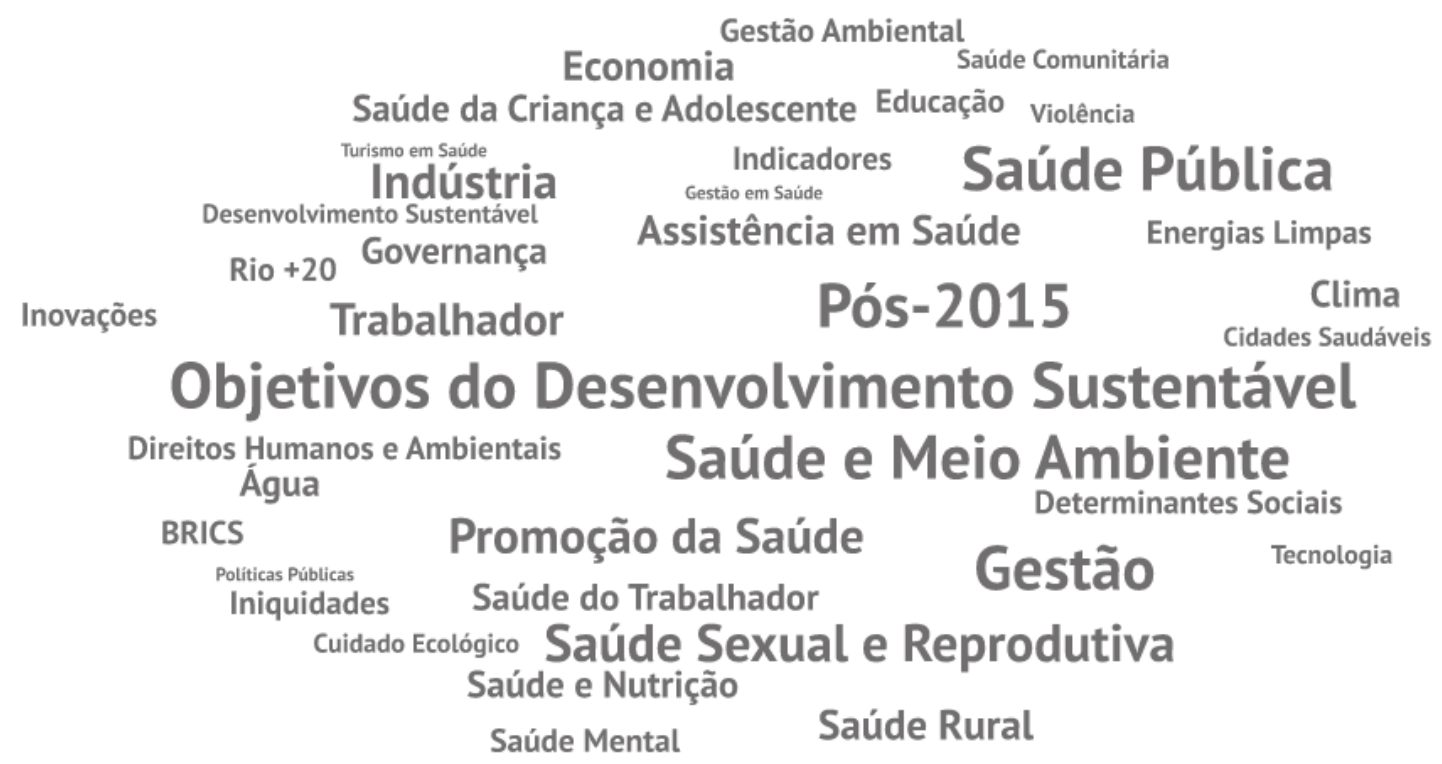

Figura 3: Nuvem de palavras dos eixos temáticos encontrados nos artigos científicos publicados, sobre saúde e desenvolvimento sustentável, entre os anos de 1992 e 2016.

Fonte: elaborado pelos autores.

$\mathrm{Na}$ análise histórica ficou evidenciado o quanto esse assunto é contemporâneo, e nos eixos temáticos as discussões sobre os ODS confirmaram mais uma vez esse fato, totalizando $13 \%$ das temáticas dos artigos publicados.

De fato, vários eventos aconteceram no planeta, atinentes às questões ambientais e sociais, como a ECO92, Agenda 21, a Convenção sobre a Biodiversidade Biológica, mas a Cúpula do Milênio, ocorrente em 2000, inspirou os ODM até 2015. Por isso, ao final da vigência dos ODM, iniciou-se a vigência dos ODS, para estabelecer as ações e esforços mundiais de desenvolvimento até 2030 (UNESCO, 2019). Dessa forma, atribui-se o aumento no número de publicações sobre esse assunto à importância mundial dessas discussões.

Do total de artigos estudados, $34,26 \%$ versou sobre desenvolvimento sustentável e saúde, destacando-se eixos como saúde e meio ambiente $(6,39 \%)$, 
saúde pública $(6,39 \%)$, saúde sexual e reprodutiva (4,65\%) e promoção da saúde $(4,07 \%)$.

A associação entre saúde e os ODM (2000-2015) foi descrita por Buss et al. (2014), os quais afirmaram que a saúde teve importante presença, constituindo três dos oito objetivos, contudo, os autores afirmaram que os demais ODM, dentre eles a sustentabilidade ambiental e estabelecimento de uma parceria mundial para o desenvolvimento também geram impacto sobre a saúde, por serem todos reconhecidos como potencialmente capazes de atuar sobre alguns dos seus principais determinantes socioambientais.

Nesse cenário, é possível observar que a saúde continua sendo objeto de atenção, conforme se pode inferir pela análise do documento final da Conferência das Nações Unidas sobre Desenvolvimento Sustentável (Rio+20), O Futuro que Queremos 2 e das iniciativas políticas globais decorrentes da orientação da Assembleia Geral das Nações Unidas de 2012, que a inclui como um dos pontos das 11 Consultas Temáticas Globais das Nações Unidas sobre os ODS.

Com relação às publicações relacionando saúde e meio ambiente (6,39\%), Corvalan et al. (2014) mencionaram como marco inicial a Conferência das Nações Unidas sobre Desenvolvimento Sustentável (Rio+20) que enfatizou em seu documento final o reconhecimento de que a saúde é um resultado e um indicador das dimensões econômica, social e ambiental do desenvolvimento sustentável.

No âmbito da saúde ambiental os estudos abordaram as condições que envolvem o ser humano e que podem exercer efeito sobre sua saúde e bemestar, com ênfase à promoção das pessoas em ecossistemas saudáveis. Boischio et al. (2009) apresentaram o conceito de ecossaúde aplicado às enfermidades transmitidas por vetores e assinalaram a necessidade de interação entre tomadores de decisões, pesquisadores e representantes da sociedade civil num processo participativo e transdisciplinar, a fim de fundamentar políticas públicas direcionadas para o desenvolvimento sustentável e saudável.

Os estudos também mostraram uma forte relação e interdependência entre desenvolvimento sustentável e a saúde pública. Os artigos em saúde pública $(6,39 \%)$, área que, tradicionalmente, possui um olhar mais voltado para a prevenção e o controle de doenças, versaram sobre os determinantes intrinsecamente relacionados à manutenção e promoção da saúde, incluindo condição social e econômica, educação, emprego, habitação e exposições físicas e ambientais. Tais fatores afetam cumulativamente a saúde de indivíduos e populações, como se observa em países com altos níveis de desemprego, baixo nível de escolaridade, moradias precárias e injustiças sociais, reduzindo a qualidade de vida (ZAMFIR et al., 2015).

Outros estudos apontam que a melhoria nos indicadores de saneamento e de educação deve ser uma prioridade, bem como, a avaliação do impacto dos programas e das políticas públicas sobre a saúde (DREWRY; KWIATKOWSKI, 
2015; GADELHA; BRAGA, 2016), de fato, Andrade et al. (2015) ressaltam a necessidade de investimento em capacidade gerencial e de compromisso político e social para sustentar o sucesso dessas políticas.

Os estudos com interesse na área de saúde infantil e adolescente confirmam essa situação. Bueno et al. (2013) analisaram os determinantes sociais da saúde através da relação entre desenvolvimento sustentável e índices de saúde infantil em população de uma região metropolitana do sul do Brasil e constataram a correlação entre indicadores de desenvolvimento sustentável associados ao resultado da saúde infantil. Os autores concluíram que as políticas sociais equitativas e sustentáveis, baseadas em estratégias inclusivas de promoção da saúde, precisam ser integradas no contexto da vida das crianças.

$\mathrm{Na}$ área da saúde e sistemas nacionais de proteção social, sob a forma de provisão de dinheiro (provisão econômica) e cuidado social (apoio psicossocial), Cluver et al. (2016) avaliaram os efeitos da provisão de proteção social sobre os indicadores relacionados à saúde entre adolescentes na África do Sul, e verificaram que a proteção social foi associada a reduções significativas de risco para adolescentes em 12 dos 17 indicadores dos ODS. Os autores concluíram que os sistemas nacionais de proteção social têm suas limitações, mas reconhecem as múltiplas e sinérgicas associações positivas com os resultados de saúde dos adolescentes.

Os estudos nacionais a respeito das ações em promoção da saúde salientam o desenvolvimento de intervenções para a melhoria do cuidado em saúde, ao reconhecer a necessidade de mais planejamento, sistematização e de incorporação das ciências sociais (PORTELA et al., 2016), comentam sobre a implantação de tecnologias sociais em comunidades vulneráveis, objetivando promover e estimular potencialidades individuais e comunitárias (OLIVEIRA et al., 2015) e compartilham a associação entre saúde e bem-estar, modelando a implementação da educação física em parcerias transversais, essenciais para o alcance dos ODS, sob a interface saúde, educação e esporte (LYNCH, 2016).

Outro eixo temático de destaque relacionado ao tema saúde foi o de saúde sexual e reprodutiva, que totalizou $4,65 \%$ das discussões. Segundo a OMS (2019), saúde sexual e reprodutiva é o estado de bem-estar físico, mental e social, em aspectos relacionados ao sistema reprodutivo em todas as fases da vida. Uma pessoa com saúde reprodutiva deve ter uma vida sexual responsável, satisfatória, segura, com capacidade de reproduzir, com informações e acesso a métodos de regulação da fertilidade de sua escolha e com direito a serviços de saúde adequados durante a gravidez e o parto.

Em nível global, durante os anos de 2005 e 2011, os ODM trouxeram maior atenção à mortalidade materna. Brolan e Hill (2013) afirmaram que a saúde e os direitos sexuais e reprodutivos exigem o engajamento de vários elementos e os defensores precisam vinculá-los aos temas-chave do desenvolvimento sustentável, como desigualdades de gênero, educação, crescimento populacional, bem como na urbanização, migração, emprego para mulheres e alterações climáticas.

revista brasileira educação ambiental 
Newman et al. (2014) sugerem que ativistas da saúde sexual e reprodutiva e dos direitos humanos devem trabalhar em parceria com os defensores do desenvolvimento sustentável e demonstrar como a saúde sexual e reprodutiva e os direitos são relevantes para estas questões. Hermann (2014) apontou que o maior desafio da atualidade é atender as necessidades das gerações atuais e futuras, sem impor pressões catastróficas ao meio ambiente. O autor reconhece que as mudanças demográficas são resultantes de escolhas pessoais e de oportunidades, e afirma que estes pontos devem ser tratados por meio de políticas que ampliem estas escolhas e oportunidades, com foco no acesso irrestrito a informações sobre saúde sexual e reprodutiva, capacitação das mulheres para participarem plenamente da vida social, econômica e política, além de investimentos na educação da nova geração.

A análise quanto à origem dos trabalhos científicos (Figura 4) indicou que pesquisadores de diferentes locais no mundo estudam o tema, porém, o Brasil se destaca com o maior número de contribuições $(21,51 \%)$, seguido pelos Estados Unidos (15,12\%) e Reino Unido (8,14\%).

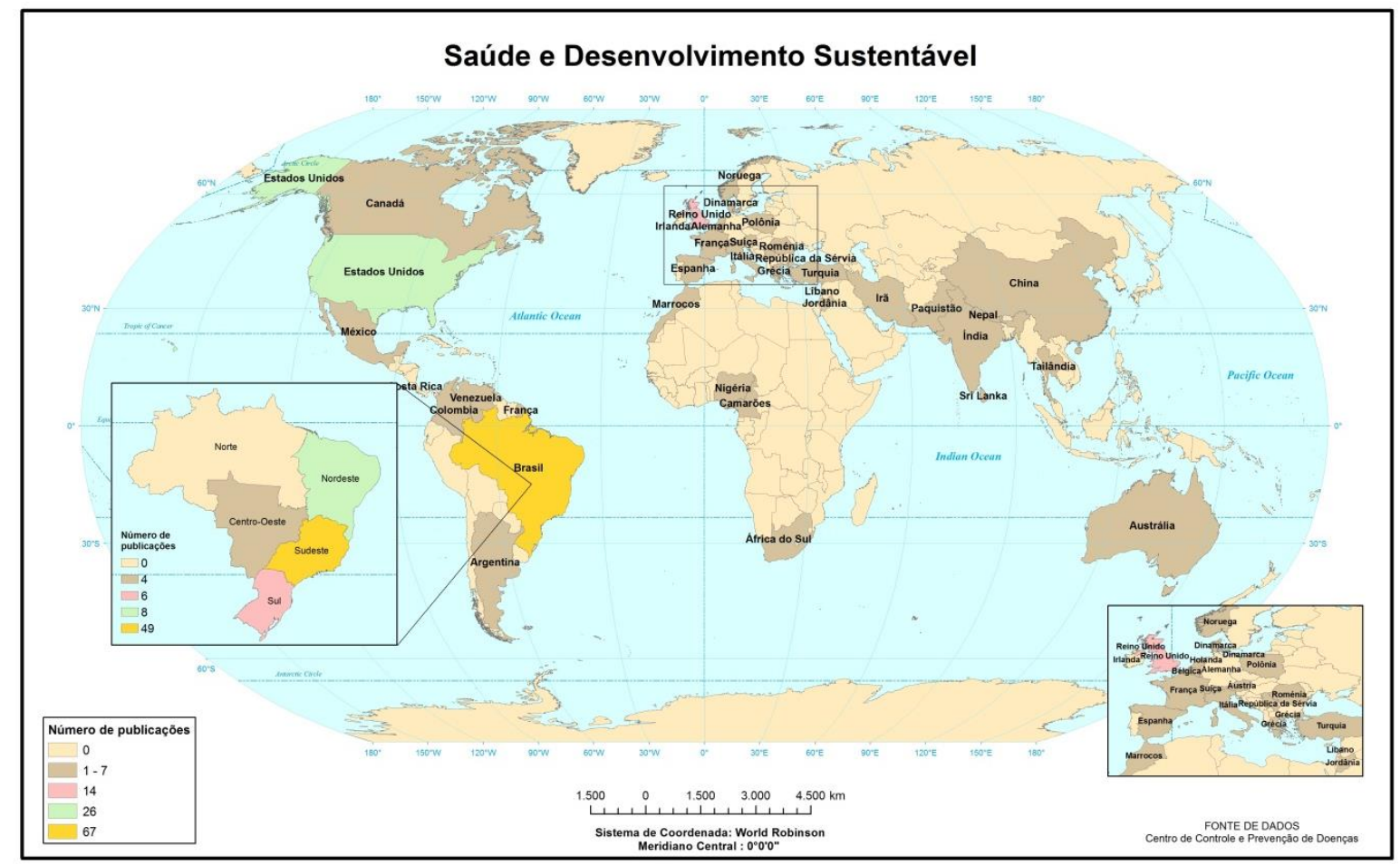

Figura 4: Distribuição geográfica dos artigos científicos publicados sobre "Saúde e Desenvolvimento Sustentável", analisados entre os anos de 1992 e 2016.

Fonte: elaborado pelos autores. 
Nações Unidas sobre Desenvolvimento Sustentável (Rio+20) e a Conferência Mundial sobre Determinantes Sociais da Saúde, ocorrida em 2011, também no Rio de Janeiro. A participação nesses eventos associada aos resultados encontrados nesta pesquisa mostram a evidente preocupação dos pesquisadores brasileiros com a busca pelo desenvolvimento sustentável e saudável.

Em função do destaque brasileiro, realizou-se um detalhamento das publicações nacionais e os resultados revelaram que os estados de Rio de Janeiro e São Paulo detiveram as maiores quantidades de publicações, 43,28\% e 16,42\%, respectivamente (Figura 4). Com efeito, esse achado reforça o mérito de instituições cariocas e paulistas, como por exemplo, Escola Nacional de Saúde Pública Sérgio Arouca da Fundação Oswaldo Cruz (RJ) e Faculdade de Saúde Pública da USP (FSP/ SP) com relação ao grande número de publicações científicas, além da forte influência do fato do Rio de Janeiro ter sediado os grandes eventos relacionados ao tema (ECO92, Rio+20).

A porcentagem de periódicos com maior concentração de publicações relacionadas à temática "Saúde e Desenvolvimento Sustentável" está representada na Figura 5. Observa-se que o periódico Ciência \& Saúde Coletiva deteve $12,2 \%$ das publicações, seguido dos periódicos Cadernos de Saúde Pública $(9,3 \%)$, Saúde e Sociedade $(8,1 \%)$ e The Lancet $(4,6 \%)$.

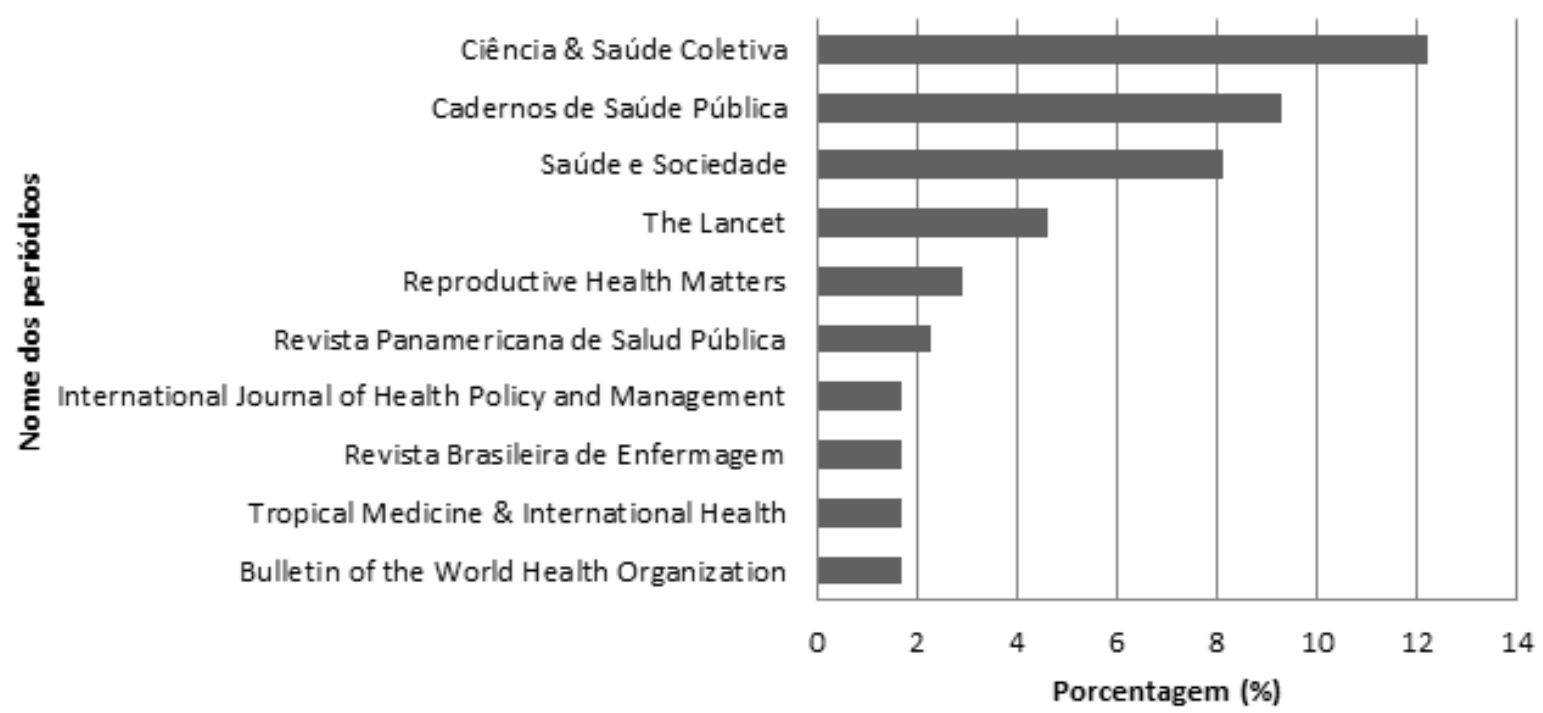

Figura 5: Periódicos científicos com maior porcentagem de artigos sobre "Saúde e Desenvolvimento Sustentável”, analisados entre os anos de 1992 e 2016.

Fonte: elaborado pelos autores.

A classificação dos periódicos de acordo o critério Qualis, instituído pela Coordenação de Aperfeiçoamento de Pessoal de Nível Superior (CAPES), fundação do Ministério de Educação e Cultura (MEC) na área de Saúde Coletiva, permite verificar que os periódicos apresentados na Figura 5 classificaram-se em estratos superiores (A1, A2 e B1), desta forma, comprova-se a alta qualidade e 
rigor científico das publicações sobre o assunto "Saúde e Desenvolvimento Sustentável" obtidas nesta pesquisa.

\section{Conclusões}

A análise histórica de 25 anos das publicações científicas sobre temas relacionados à saúde e desenvolvimento sustentável permitiu observar um número reduzido de publicações no período, sinalizando uma lacuna científica preocupante.

Contudo, a partir de 2012, sustentados pelas discussões dos ODM e ODS, o número de publicações aumentou substancialmente e versaram sobre diferentes eixos, sobretudo relacionados à saúde e meio ambiente, saúde pública e gestão, porém, temas como segurança alimentar, agricultura sustentável, acesso à energia, cidades sustentáveis e saudáveis, sociedades pacíficas e inclusivas e educação ambiental, que notoriamente devem se articular com as ações em saúde, foram escassos.

O Brasil se destacou como o país com o maior número de publicações sobre $o$ assunto, sendo que este fato resulta, em grande parte, das pesquisas desenvolvidas nos estados do Rio de Janeiro e São Paulo.

Os resultados deste estudo trazem um panorama profícuo para geração, difusão e aplicação do conhecimento técnico e científico na interface saúde e desenvolvimento sustentável, pois identificou vulnerabilidades e potencialidades para o alcance dos ODS e para a relação segura entre o desenvolvimento sustentável e a saúde dos seres humanos.

\section{Agradecimentos}

À Coordenação de Aperfeiçoamento de Pessoal de Nível Superior (CAPES) e Instituto Cesumar de Ciência, Tecnologia e Inovação (ICETI), pelo apoio financeiro à pesquisa. 


\section{Referências}

ABOUETTAHIR, R.; SEGHIOUER, H.; EL AMARTI, A. Occupational health and safety in a sustainable development approach: The case of Moroccan companies. Archives des Maladies Professionnelles et de l'environnement. v.74, n.4, p.380-386, 2013.

ALLI, B.; BROWN, B.; BAKER, G.; WARBER, J. The indaba declaration on food, nutrition, health and sustainable development. Public Health Nutrition. v.5, n.6, p.711-713, 2002.

ANDRADE, L. O.; PELLEGRINI FILHO, A.; SOLAR, O.; RÍGOLI, F.; SALAZAR, L. M.; SERRATE, P. C.; RIBEIRO, K. G.; KOLLER, T. S.; CRUZ, F. N; ATUN, R. Social determinants of health, universal health coverage, and sustainable development: case studies from Latin American countries. The Lancet. v. 385, n. 9975, p.1343-1351, 2015.

BACKES, M. T. S.; BACKES, D. S.; DRAGO, L. C.; KOERICH, M. S.; ERDMANN, A. L. Cuidado ecológico como um fenômeno amplo e complexo. Revista Brasileira de Enfermagem. v. 64, n.5, p. 876-81, 2011.

BERMAN, P. Health sector reform: making health development sustainable. Health Policy. v.32, n.1, p. 13-28, 1995.

BOISCHIO, A.; SÁNCHEZ, A.; OROSZ, Z.; CHARRON, D. Health and sustainable development: challenges and opportunities of ecosystem approaches in the prevention and control of dengue and Chagas disease. Cadernos de Saúde Pública, v. 25, n.11, p.149-154, 2009.

BORGES, A. M.; BONOW, C. A.; SILVA, M. R. S.; ROCHA, L. P.; CEZAR-VAZ, M. R. Agricultura familiar ea conservação da saúde humana e ambiental. Revista Brasileira de Enfermagem. v.9, n.2, p. 326-334, 2016.

BOS, R. New approaches to disease vector control in the context of sustainable development. Cadernos de Saúde Pública. v.3, p. 240-248, 1992.

BOTCHWEY, N. D.; TROWBRIDGE, M.; FISHER, T. Green health: urban planning and the development of healthy and sustainable neighborhoods and schools. Journal of Planning Education and Research. v.34, n.2, p. 113-122, 2014.

BROLAN, C. E.; HILL, P. S. Countdown for health to the post-2015 UN Sustainable Development Goals. Medical Journal of Australia. v.202, n. 6, p.289-90, 2015.

BRUNDTLAND, G. H. Health and the world conference on sustainable development. Bulletin of the World Health Organization. v.80, n.9, p.689-690, 2002.

BUENO, R.E.; MOYSÉS, S.; BUENO, P.A.R.; MOYSÉS, S.J.; CARVALHO, M.L.; FRANÇA, B.H.S. Sustainable development and child health in the Curitiba metropolitan mesoregion, State of Paraná, Brazil. Health \& Place, v.19, p.167173, 2013. 
BUNDHAMCHAROEN, K.; LIMWATTANANON, S.; KUSREESAKUL, K.; TANGCHAROENSATHIEN, V. Contributions of national and global health estimates to monitoring health-related sustainable development goals. Global Health Action. v.9, n.1, p. 32243-32251, 2016.

BUSS, P. M.; MAGALHÃES, D. P.; SETTI, A. F. F.; GALLO, E.; FRANCO NETTO, F. A.; MACHADO, J. M. H.'BUSS, D. F. Saúde na agenda de desenvolvimento pós-2015 das Nações Unidas. Cadernos de Saúde Pública. v.30, n.12, p. 2555-2570, 2014.

CLUVER, Lucie D. et al. Can social protection improve sustainable development goals for adolescent health? PLoS One, v.11, n.10, p. e0164808, 2016.

CARNEIRO, F. F.; FRANCO NETTO, G.; CORVALAN, C.; FREITAS, C.M.; SALES, L.B. Environmental health and inequalities: building indicators for sustainable development. Ciência \& Saúde Coletiva, v. 17, n. 6, p. 1419-1425, 2012.

CHAPMAN, A. R. Assessing the universal health coverage target in the Sustainable Development Goals from a human rights perspective. BMC International Health and Human Rights. v.16, n.1, p. 33-41, 2016.

CLUVER, L.D.; ORKIN, F.E.; MEINCK, F.; BOYES, M.E.; YAKUBOVICH, A.R.; SHERR, L. Can social protection improve sustainable development goals for adolescent health? PLoS One, v.11, n.10, p. e0164808, 2016.

COMISSÃO MUNDIAL SOBRE O MEIO AMBIENTE E DESENVOLVIMENTO CMMAD. Nosso futuro Comum. 2.ed. Rio de Janeiro: Fundação Getúlio Vargas, $1991 . \quad$ Disponível em $<$ http://www.scielo.br/scielo.php?script=sci nlinks\&ref=000219\&pid=S00347612201400010000900009\&lng=pt>, acesso em jan. 2019.

CORVALAN, C.; DUARTE, E. C.; VAZQUEZ, E. Desenvolvimento sustentável e saúde: tendências dos indicadores e desigualdades no Brasil. $1^{\circ} \mathrm{ed}$. Distrito Federal: OPAS, 2014, 30p.

DELGADO, I. J. G. Healthcare systems, the State, and innovation in the pharmaceutical industry. Cadernos de Saúde Pública. v.32, n.2, p.1-13, 2016.

DESSEILLES, M.; DUCLOS, C.; FLOHIMONT, V.; DESSEILLES, F. Is there a role for "climatotherapy" in the sustainable development of mental health?. Behavioral and Brain Sciences. v.36, n.5, p. 487-488, 2013.

DREWRY, J.; KWIATKOWSKI, R. The role of health impact assessment in advancing sustainable development in Latin America and the Caribbean. Journal of Environmental Health. v. 77, n.8, p.16-20, 2015.

FURUKAWA, P. O.; CUNHA, I. C. K. O.; PEDREIRA, M. L. G. Avaliação de ações ecologicamente sustentáveis no processo de medicação. Revista Brasileira de Enfermagem. v. 69, n.1, p.23-29, 2016.

GADELHA, C. A.G.; BRAGA, P.S. C. Health and innovation: economic dynamics and Welfare State in Brazil. Cadernos de Saúde Pública. v.32, 2016. 
GALLO, E.; SETTI A. F. F.; MAGALHÃES, D. P.; MACHADO, J. M. H.; BUSS, D. F.; FRANCO NETTO, F. A.; BUSS, P. M. Saúde e economia verde: desafios para o desenvolvimento sustentável e erradicação da pobreza. Ciência \& Saúde Coletiva. v.17, n.6, p.1457-1468, 2012.

GALVÃO, L. A. C.; HABY, M. M.; CHAPMN, E.; CLARK, R.; CÂMARA, V. M.; LUIZ, R. R.; BECERRA-POSADA, F. The new United Nations approach to sustainable development post-2015: Findings from four overviews of systematic reviews on interventions for sustainable development and health. Revista Panamericana de Salud Pública. v.39, n.3, p.157-165, 2016.

GIDDINGS, B.; HOPWOOD, B.; O'BRIEN, G. Environment, economy and society: fitting them together into sustainable development. Sustainable Development. v.10, n.4, p.187-196, 2002.

GROVE, J.; CLAESON, M.; BRYCE, J.; AMOUZOU, A.; BOERMA, T.; WAISWA, P.; VICTORA, C. Maternal, newborn, and child health and the Sustainable Development Goals-a call for sustained and improved measurement. The Lancet. v.386, n.10003, p. 1511-1514, 2015.

HELLEN, J. Tourist health and tourist medicine in the tropics: A case for sustainable development. The health of nations. Tourist Studies. v.1, n.3, p.153-176, 1995.

HERRMANN, M. Sustainable development, demography and sexual and reproductive health: inseparable linkages and their policy implications. Reproductive Health Matters, v. 22, n.43, p.28-42, 2014.

INTERGOVERNMENTAL PANEL ON CLIMATE CHANGE - IPCC. Climate Change. Paris. 2007. Disponível em $<$ http://www.ipcc.ch/publications and data/ar4/wg3/en/contents.html> acesso em: abr. 2019.

JOHN, T. J.; ABEL, R. Sustainable development and health: an Indian perspective. The Lancet. v.360, n.9333, p.638-639, 2002.

KHETANI, M.; MARLEY, J.; BAKER, M.; ALBRECHT, E.; BEDELL, G.; COSTER, W.; ANABY, D.; LAW, M. Validity of the Participation and Environment Measure for Children and Youth (PEM-CY) for Health Impact Assessment (HIA) in sustainable development projects. Disability and Health Journal. v.7, n.2, p. 226-235, 2014.

KIM, J. Y.; EVANS, T. Promoting women's health for sustainable development. The Lancet. v.386, n.9999, p.9-10, 2015.

LYNCH, T. United Nations Sustainable Development Goals: Promoting health and well-being through physical education partnerships. Cogent Education, v. 3, n.1, 2016.

MARTENS, W. J.; SLOOFF, R.; JACKSON, E. K. Climate change, human health, and sustainable development. Bulletin of the World Health Organization. v.75, n.6, p. 583-584, 1997. 
MELO, M. A. B. C.; COSTA, N. R. Sustainable development, structural adjustment and social policy: the strategies of WHO/PAHO and the World Bank for Health Care. Planejamento e Políticas Públicas. v.1, n.1, p.61-64, 1994.

NEWMAN, K.; FISHER, S.; MAYHEW, S.; STEPHENSON, J. Population, sexual and reproductive health, rights and sustainable development: forging a common agenda. Reproductive Health Matters. v.22, n.43, p.53-64, 2014.

OLIVEIRA, E. F.; JESUS, V. S.; SIQUEIRA, S. M. C.; ALVES, T. A.; SANTOS, I.M.; CAMARGO, C.L. Promoting health in vulnerable communities: social technologies for poverty reduction and sustainable development. Revista Gaúcha de Enfermagem, v. 36, n. SPE, p. 200-206, 2015.

ORGANIZAÇÃO DAS NAÇÕES UNIDAS PARA A EDUCAÇÃO A CIÊNCIA E A CULTURA - UNESCO - Agenda de Desenvolvimento pós-2015 - UNESCO e os Objetivos de Desenvolvimento Sustentável. Disponível em $<$ http://www.unesco.org/new/pt/brasilia/post-2015-development-agenda>. acesso em: jan. 2019.

ORGANIZAÇÃO DAS NAÇÕES UNIDAS PARA A EDUCAÇÃO A CIÊNCIA E A CULTURA - UNESCO - Agenda de Desenvolvimento pós-2015 - UNESCO e os Objetivos de Desenvolvimento Sustentável. Disponível em $<$ http://www.unesco.org/new/pt/brasilia/post-2015-development-agenda/>, acessado em 08 de março de 2019.

ORGANIZAÇÃO MUNDIAL DA SAÚDE - OMS. Sexual and reproductive health. Disponível em <https://www.who.int/reproductivehealth/en/> acesso em jan. 2020.

PADILHA, N. S. Fundamentos Constitucionais do Direito Ambiental Brasileiro. 1ed. Rio de Janeiro: Editora Elsevier, 2010, 452p.

PEIRY, K. K. Public health in international law: the contribution of the World Summit on Sustainable Development. Bulletin of the World Health Organization, v.80, n.12, p.925-925, 2002.

PORTELA, M. C.; LIMA, S. M. L.; MARTINS, MÔNICA, T. C. Improvement Science: conceptual and theoretical foundations for its application to healthcare quality improvement. Cadernos de Saúde Pública, 32 (Suppl 2), p.e00105815, 2016.

PRICE, C.; TSOUROS, A. Our cities, our future: policies and action plans for health and sustainable development. Environment and Urbanization, v.1, n.1, p. 266-270, 1997.

RIBEIRO, H.; GÜNTHER, W. M. R.; ARAÚJO, J. M. Avaliação qualitativa e participativa de projetos: uma experiência a partir de pesquisa em educação ambiental e saneamento do meio. Saúde e Sociedade. v.11, n.2, p. 107-132, 2002. 
RIBEIRO, P. J. M.; BARCELLOS, C.; ROQUE, O. C. C. Development challenges in Miracema (RJ, Brasil): a territorial approach for the sustainable management of health and environment. Saúde e Sociedade. v.22, n.2, p.575-589, 2013.

SIGNORINO, G. Sustainable Development: From Economy to Environment and Health. Measuring the Sustainability of Industrialization Patterns Through ExPost Sanitary Evaluations: The Petrochemical Areas in Sicily. Epidemiology, v.22, n.1, p.120-125, 2011.

TOMLINSON, M. Global mental health: a sustainable post Millennium Development Goal? International Health. v.5, n.1, p.1-3, 2013.

VALENTINE, N. B.; KOLLER, T. S.; HOSSEINPOOR, A. R. Monitoring health determinants with an equity focus: a key role in addressing social determinants, universal health coverage, and advancing the 2030 sustainable development agenda. Global Health Action. v.9, n.1, p.1-7, 2016.

VAN LOON, T. Sustainable Development In The Prevention Of Sexual Delinquency-Public Health And Security. WIT Transactions on Ecology and the Environment. v.54, n.1, p. 771-779, 2002.

VORSTER, H. H. E. The 2002 World Summit on Sustainable Development: A more holistic approach for public health nutrition? Public Health Nutrition. v.5, n.6, p.707-708, 2002.

WARFORD, J. J. Environment, health, and sustainable development: the role of economic instruments and policies. Bulletin of the World Health Organization. v.73, n.3, p.387-388, 1995.

WATERSTON, T., LENTON, S. Sustainable development, human induced global climate change, and the health of children. Archives of Disease in Childhood. v.82, n.2, p.95-97, 2000. 